\title{
High-accuracy long-distance measurements in air with a frequency comb laser
}

\author{
M. Cui, ${ }^{1, *}$ M. G. Zeitouny, ${ }^{1}$ N. Bhattacharya, ${ }^{1}$ S. A. van den Berg, ${ }^{2}$ H. P. Urbach, ${ }^{1}$ and J. J. M. Braat ${ }^{1}$ \\ ${ }^{1}$ Department of Applied Sciences, Optics Research Group, Technical University Delft, Lorentzweg 1, \\ 2628 CJ Delft, The Netherlands \\ ${ }^{2}$ Van Swinden Laboratorium, Thijsseweg 11, 2629 JA Delft, The Netherlands \\ *Corresponding author: m.cui@tudelft.nl
}

Received April 24, 2009; accepted May 17, 2009;

posted May 28, 2009 (Doc. ID 110571); published June 24, 2009

We experimentally demonstrate that a femtosecond frequency comb laser can be applied as a tool for longdistance measurement in air. Our method is based on the measurement of cross correlation between individual pulses in a Michelson interferometer. From the position of the correlation functions, distances of up to $50 \mathrm{~m}$ have been measured. We have compared this measurement to a counting laser interferometer, showing an agreement with the measured distance within $2 \mu \mathrm{m}\left(4 \times 10^{-8}\right.$ at $\left.50 \mathrm{~m}\right)$. () 2009 Optical Society of America

OCIS codes: $320.7100,320.2250,320.1590,120.3930$.

Traditional techniques for long-distance measurements are often based on optical interferometry when the demands on accuracy rise. Most of these interferometric techniques rely on incremental measurements of phase accumulation. A priori knowledge of the distance to be measured is required or a complex multiwavelength system may be needed. In 2004, Ye [1] proposed a simple scheme for measuring long distances in space with a stabilized femtosecond frequency comb. The scheme is based on a Michelsontype interferometry with optical interference between individual pulses. The technique proposed by $\mathrm{Ye}$ has been demonstrated for interferometric measurement of short displacement $[2,3]$. The main advantage of applying a frequency comb for distance measurement is the large range of nonambiguity, which is determined by the cavity length of the pulsed laser, ranging from about $30 \mathrm{~cm}$ to $3 \mathrm{~m}$. It is thus not necessary to rely on incremental measurement of the optical phase. The ambiguity is easily overcome by, e.g., a laser distance meter. The stabilized frequency comb has been applied as a source in various distance measurement schemes $[4,5]$. In this Letter, we demonstrate distance measurements of up to $50 \mathrm{~m}$ in air by analyzing the cross correlation between pulses emitted from a stabilized frequency comb source. We have implemented a model of pulse propagation in air to account for the effect of air dispersion on the measured cross-correlation functions. The measurement results obtained with the frequency comb and a conventional counting laser interferometer are compared.

A mode-locked Ti:sapphire laser is the frequency comb source, with both the repetition frequency and the carrier-envelop offset (CEO) frequency referenced to a cesium atomic clock (Fig. 1). The pulse duration is $40 \mathrm{fs}$, and the repetition rate $f_{r}$ is locked at approximately $1 \mathrm{GHz}$, corresponding to a pulse to pulse distance $l_{\mathrm{pp}}=c /\left(n_{g} f_{r}\right)$ of $30 \mathrm{~cm}$. Here $c$ is the speed of light in vacuum and $n_{g}$ is the group refractive index at the center wavelength. The CEO frequency $f_{0}$ is fixed at $180 \mathrm{MHz}$. The center wavelength of the pulses is $815 \mathrm{~nm}$, with an FWHM of about $20 \mathrm{~nm}$. Af- ter collimation the beam is sent to a Michelson interferometer. One part of the beam is reflected by a hollow corner cube mounted on a piezoelectric transducer (PZT) along the short reference arm. The length of the short arm can be scanned by a translation stage. The other part of the beam is reflected by two mirrors and propagates along the long measurement arm. One of the mirrors is coated for high transmission at $633 \mathrm{~nm}$ for comparison with a helium-neon (He-Ne) laser interferometer. The long measurement arm consists of a hollow corner cube mounted on a mechanical car that can move along a bench of $50 \mathrm{~m}$. A parallel plate is used in the long arm to compensate the dispersion of the plate beam splitter. The returning beams are overlapped and focused on an avalanche photodiode (APD). A coherence maximum appears when the total path length difference between both arms is a multiple of $l_{\text {pp }}$. Firstorder cross-correlation functions are then obtained

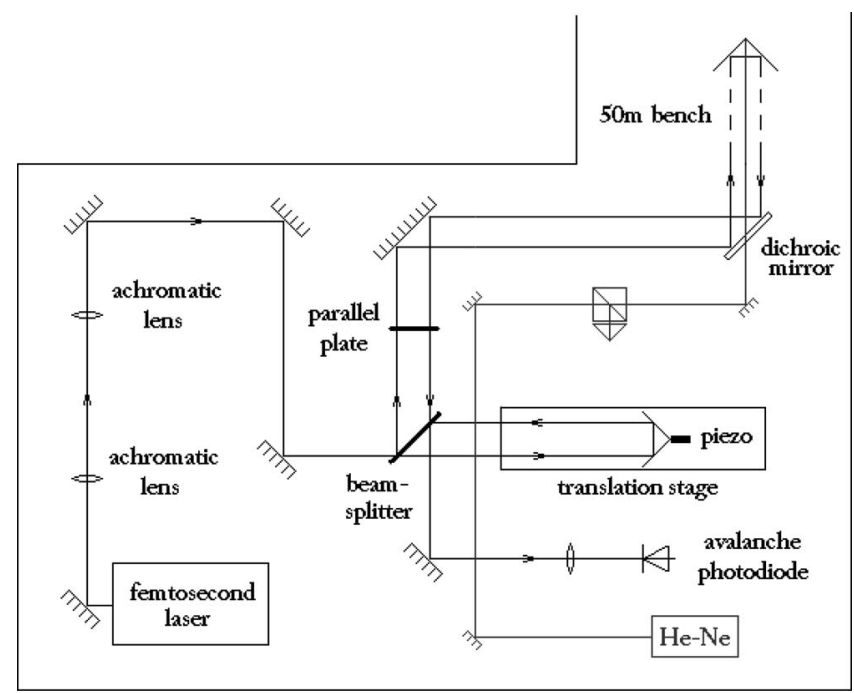

Fig. 1. Schematic of the experimental setup. The collimated pulsed beam from a Ti:sapphire-laser-based frequency comb is sent to a Michelson interferometer. A calibrated $\mathrm{He}-\mathrm{Ne}$ laser interferometer is used for an independent comparison measurement. 
by modulating the PZT at $50 \mathrm{~Hz}$ with a sine modulation within a range of $80 \mu \mathrm{m}$. The beam of a He-Ne laser interferometer copropagates with the frequency comb and measures the displacement of the long arm independently.

The measurement is carried out by first placing the mechanical car with the retroreflector in the long arm at the closest possible position to the dichroic mirror. At this position the path length difference between both arms is $0.9 \mathrm{~m}$. The cross-correlation pattern is located by moving the translation stage and recorded by scanning the PZT. Subsequently the mechanical car is moved over a distance of about $10 \mathrm{~m}$ (corresponding to a path length change of $68 l_{\mathrm{pp}}$ ), such that another cross-correlation pattern is located and recorded. At each position five correlation patterns have been recorded and the entire procedure has been repeated six times. Similar measurements have been done by moving the mechanical car over 20,30 , 40 , and $50 \mathrm{~m}$ (corresponding to path length changes of $136,204,272$, and 339 times the pulse to pulse distance, respectively).

Considerable broadening and chirp are observed in the correlation patterns because of the dispersion of the pulse in air. To determine the effect of dispersion on the measured distance, a numerical model based on plane-wave propagation has been developed. Assuming that the environmental conditions are identical in both arms and the intensities of the two beams are equal, we can write

$$
\begin{aligned}
& E_{1}\left(t, d_{1}\right)=\sum a_{m} e^{i\left(2 \pi f_{m} t-k_{m} d_{1}\right)}, \\
& E_{2}\left(t, d_{2}\right)=\sum a_{m} e^{i\left(2 \pi f_{m} t-k_{m} d_{2}\right)} .
\end{aligned}
$$

Here $k_{m}=2 \pi n\left(\lambda_{m}\right) / \lambda_{m}$ and $n\left(\lambda_{m}\right)$ is the refractive index of air at the wavelength $\lambda_{m}$, which can be determined by the updated Edlén equation [6]. The pulses propagating along the two arms of the interferometer travel distances $d_{1}$ and $d_{2}$, respectively. The range of the integer $m$ is chosen such that all contributing frequency comb modes (with frequencies of $f_{m}=f_{0}+m f_{r}$ ) are taken into account. The intensity can be written as

$$
\left\langle I\left(d_{1}, d_{2}\right)\right\rangle=\left\langle\left(E_{1}+E_{2}\right)\left(E_{1}+E_{2}\right)^{*}\right\rangle,
$$

where $E_{1}$ and $E_{2}$ are complex and $I$ is always nonnegative. Therefore we get

$$
\left\langle I\left(d_{1}, d_{2}\right)\right\rangle=2 \sum_{m=1}^{M}\left|a_{m}\right|^{2}\left(1+\cos \left[k_{m}\left(d_{1}-d_{2}\right)\right]\right) .
$$

The spectral content of the pulse has been extracted from an autocorrelation measurement at zero path length difference. The results have been compared with an independent measurement made with an Ocean Optics spectrometer (Fig. 2). The spectrum has a maximum at $815 \mathrm{~nm}$ and an FWHM of $22 \mathrm{~nm}$. The spectrum obtained from the autocorrelation measurement has been used to determine the pulse propagation according to Eq. (2).

The experimentally measured cross correlations compared with our numerical model are shown in
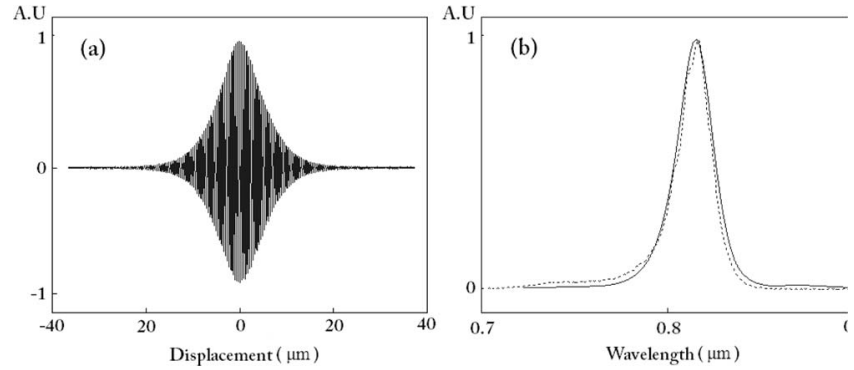

Fig. 2. (a) Autocorrelation at equal arms. (b) Spectrum derived from the autocorrelation (solid curve) compared with the spectrum measured using an Ocean Optics spectrometer (dotted curve).

Fig. 3. The amplitude of the cross-correlation function drops to $30 \%$ after $50 \mathrm{~m}$ propagation in air. This decrease in amplitude can be explained by the divergence of the beam and the fact that only some parts of the beam reflected back impinges on the APD. The experimental and numerical correlation patterns show good agreement in shape and broadening.

The measured distance has been extracted from the position of the maximum of the cross correlations, which is found by curve fitting the envelope with a Gaussian. Owing to group velocity dispersion in air, the position of maximum coherence, i.e., the peak of the correlation function at different positions, does not exactly overlap with a multiple of $l_{\mathrm{pp}}$, which is calculated from $f_{r}$ and the group refractive index at the maximum wavelength of the spectrum. The distance that the peak of the correlation pattern is shifted is given by $d_{\text {chirp }}$. This value depends on the spectral content and the distance traveled. For distances of up to $50 \mathrm{~m}$, the value varies from 0.5 to $0.8 \mu \mathrm{m}$ as shown in Table 1 .

The distance measured is then given by
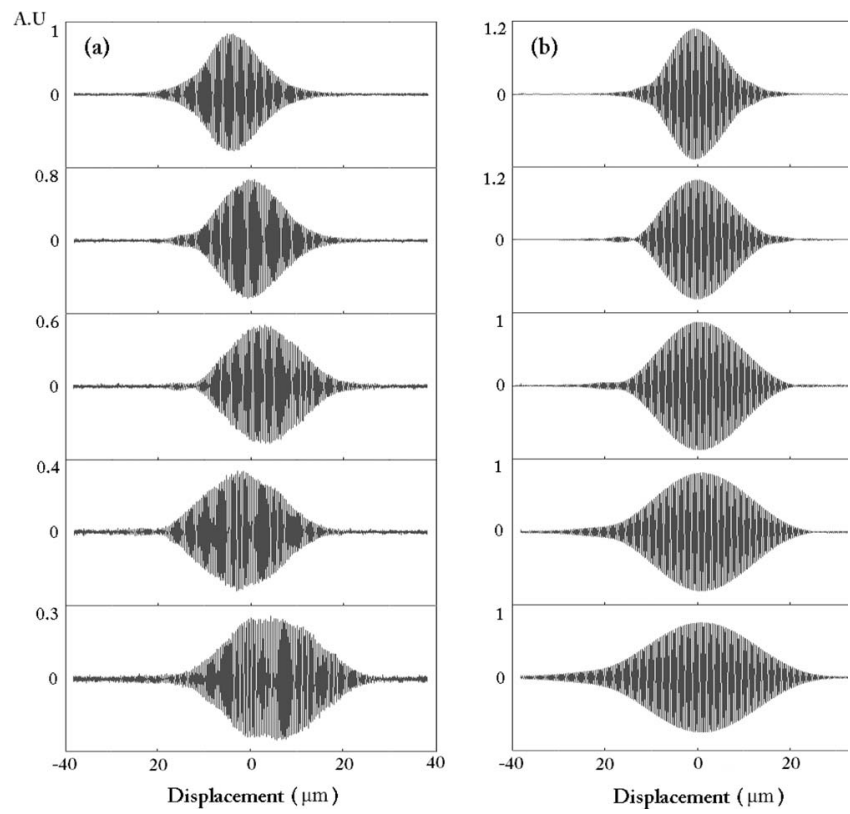

Fig. 3. Cross correlations at different positions. (a) Measured cross correlations. (b) Cross correlations from the numerical model. 
Table 1. Shift of the Correlation Patterns Owing to the Chirp at Different Lengths

\begin{tabular}{lccccc}
\hline & $10 \mathrm{~m}$ & $20 \mathrm{~m}$ & $30 \mathrm{~m}$ & $40 \mathrm{~m}$ & $50 \mathrm{~m}$ \\
\hline$d_{\text {chirp }}(\mu \mathrm{m})$ & 0.56 & 0.73 & 0.76 & 0.77 & 0.75 \\
\hline & $l_{\text {meas }}=p l_{\mathrm{pp}} / 2+d_{\text {chirp }}+\delta$, & & $(4)$
\end{tabular}

with $p$ being an integer number. For each distance the maxima of the correlation patterns before and after the movement were located by fitting Gaussian profiles through them. Since the displacement can only approximately be set to a multiple of $l_{\mathrm{pp}}$, the peaks do not appear at exactly the same PZT position (as can also be seen in Fig. 3). This is taken into account by the calibration of the PZT. From the relative peak position the correction term $\delta$ is obtained and added in Eq. (4), such that the real displacement is retrieved.

Figure 4 shows the results obtained as compared with a fringe counting wavelength calibrated $\mathrm{He}-\mathrm{Ne}$ laser. Each data point has been averaged from six independent measurements for both lasers. From the figure, it can be seen that the measurements using the frequency comb laser agree with the reference measurement done with the $\mathrm{He}-\mathrm{Ne}$ laser within $2 \mu \mathrm{m}$.

During the measurement, the temperature, the pressure, and the humidity have been monitored. The variation and the uncertainty on the measurement of these environmental parameters limit the accuracy in measuring the absolute distances. For example, a typical uncertainty of $0.2^{\circ} \mathrm{C}$ in temperature or $0.5 \mathrm{hPa}$ in pressure already leads to an uncertainty of the refractive index of air of about $5 \times 10^{-7}$, which corresponds to $25 \mu \mathrm{m}$ at $50 \mathrm{~m}$. Moreover the updated Edlén equation itself has an intrinsic uncertainty of $3 \times 10^{-8}$. The influences of the environmental parameters on the refractive index of the wavelengths at both the He-Ne laser and the frequency comb cancel in the first order. This is the reason why the agreement between the experimental results is much better than $25 \mu \mathrm{m}$. We attribute the residual difference and the uncertainty of the comparison measurement to vibrations in the setup and air turbulence. The agreement between the frequency comb and the He-Ne laser is much better than the achievable absolute accuracy in air, showing that the measurement result is not limited by the method chosen.

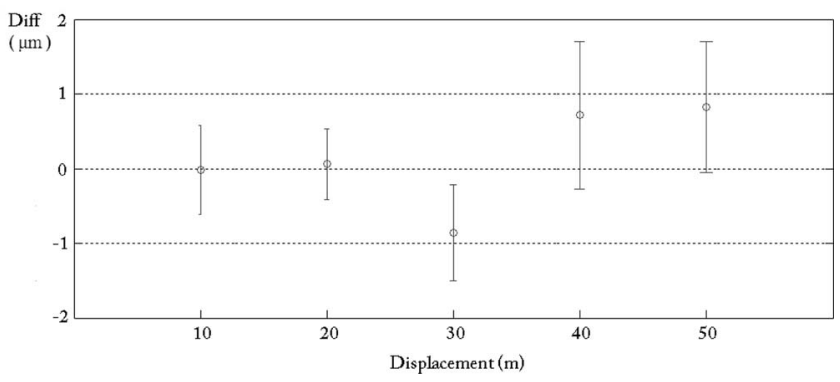

Fig. 4. Measurement at displacements of around 10, 20, 30,40 , and $50 \mathrm{~m}$. The error bars indicate the standard uncertainty derived from measurement reproducibility.

In conclusion, we demonstrated that the frequency comb is a suitable source for distance measurement. In our experiment, we fixed the position of the reference arm and moved the measurement arm close to a multiple of the cavity length. The method can be generalized for measuring arbitrary distances. In this case the short arm needs to be moved over a calibrated distance to obtain the correlation. The frequency comb has the advantage of a large nonambiguity range and depending on the linewidth of the particular comb source, coherence lengths of tens of kilometers or more are feasible. This is a good property to expect that this method can be applied to measure very long distances, especially in space where the dispersion of air is absent.

The authors thank P. Balling and S. Persijn for discussions. This research is funded by the Stichting voor Fundamenteel Onderzoek der Materie (FOM). This research lies within the European Association of National Metrology Institutes and is funded by the European Networking of National or Regional Programs Plus under grant agreement 217257.

\section{References}

1. J. Ye, Opt. Lett. 29, 1153 (2004).

2. M. Cui, R. N. Schouten, N. Bhattacharya, and S. A. van den Berg, J. Eur. Opt. Soc. Rapid Publ. 3, 08003 (2008).

3. P. Balling, P. Křen, P. Mašika, and S. A. van den Berg, Opt. Express 17, 9300 (2009).

4. Y. Salvade, N. Schuhler, S. Leveque, and S. Le Floch, Appl. Opt. 47, 2715 (2008), and references therein.

5. K. N. Joo, Y. Kim, and S. W. Kim, Opt. Express 16, 19799 (2008), and references therein.

6. K. P. Birch and M. J. Downs, Metrologia 31, 315 (1994). 